\title{
Soot Particle Size Distribution Functions in a Turbulent Non-Premixed Ethylene-Nitrogen Flame
}

\author{
Wesley Boyette $^{1}$. Snehaunshu Chowdhury ${ }^{1}$. \\ William Roberts ${ }^{1}$
}

Received: date / Accepted: date

\begin{abstract}
A scanning mobility particle sizer with a nano differential mobility analyzer was used to measure nanoparticle size distribution functions in a turbulent non-premixed flame. The burner utilizes a premixed pilot flame which anchors a $\mathrm{C}_{2} \mathrm{H}_{4} / \mathrm{N}_{2}(35 / 65)$ central jet with $\operatorname{Re}_{D}=20,000$. Nanoparticles in the flame were sampled through a $\mathrm{N}_{2}$-filled tube with a $500-\mu \mathrm{m}$ orifice. Previous studies have shown that insufficient dilution of the nanoparticles can lead to coagulation in the sampling line and skewed particle size distribution functions. A system of mass flow controllers and valves were used to vary the dilution ratio. Singlestage and two-stage dilution systems were investigated. A parametric study on the effect of the dilution ratio on the observed particle size distribution function indicates that particle coagulation in the sampling line can be eliminated using a two-stage dilution process. Carbonaceous nanoparticle (soot) concentration particle size distribution functions along the flame centerline at multiple heights in the flame are presented. The resulting distributions reveal a pattern of increasing mean particle diameters as the distance from the nozzle along the centerline increases.
\end{abstract}

Keywords Turbulent non-premixed flames - Soot · Particle size distribution function . Scanning mobility particle sizer · Differential mobility analyzer

\section{Introduction}

Ultrafine soot particles generated from combustion are known to be harmful to the environment [1] and create serious public health concerns [2]. These particles are easily transported over long distances by air currents [3] and can adversely affect the local radiative properties of air [4], contributing to global warming. Deposition of these ultrafine particles in the lungs through inhalation could result in chronic respiratory illnesses [5]. The presence of soot is ubiqitous in many processes that burn fossil fuels and an understanding of the physical processes that lead to its formation is critical to mitigating the environmental and public health concerns that come with it [6].

Wesley Boyette

Clean Combustion Research Center (CCRC), King Abdullah University of Science and Technology (KAUST), Thuwal, 23955-6900, Saudi Arabia

E-mail: wesley.boyette@kaust.edu.sa 
A number of diagnostic techniques for detecting soot exist. These diagnostics tend to focus on measuring the total soot volume fraction and/or the soot particle size distribution. D'Anna gives an overview of the pros and cons of the most common techniques, including intrusive techniques such as scanning mobility particle sizing (SMPS, also called differential mobility analysis), transmission electron microscopy (TEM), and atomic force microscopy (AFM), as well as non-intrusive techniques like laser-induced incandescence (LII), laserinduced fluorescence (LIF), and scattering/extinction [7]. SMPS has been used extensively over the last 20 years to characterize the nanoparticle size distribution in laminar sooting flames. While this technique requires careful implementation to get accurate results, it has the benefit of being able to measure size distributions from $1 \mathrm{~nm}$ up to over $100 \mathrm{~nm}$ without any manipulation of the sample. One laminar premixed flame that has been of particular interest is composed of a rich mixture of ethylene, argon, and oxygen. Zhao et al. found that the dilution ratio (the ratio of volume of nitrogen in the sampling line to the volume extracted from the flame) was a critical factor in eliminating the effects of particle coagulation in the sampling line [8]. In another paper from the same group, it was estimated that a critical dilution ratio of $10^{4}$ was necessary [9]. A study published in 2005 found that the particle size distribution was bimodal at low temperatures but then transitioned to a unimodal shape at higher temperatures; a feature that could be explained by competition between particle nucleation and particle-particle coagulation [10]. A later report indicated that the location of the bimodal split in the particle size distribution function was dependent on flame temperature and that the presence of bimodality was not necessarily indicative of particle aggregation [11]. By comparing results from SMPS, TEM, and small angle neutron scattering, discrepancies between soot measurements made by SMPS and TEM were attributed to the fact that soot particles formed in this flame were liquid-like and thus tend to deform when deposited on a TEM grid [12]. In all of these cases, a single-stage dilution probe was used. Maricq et al. demonstrated the use of a three-stage ejector pump to sample from a rich premixed ethylene-air flat flame and the results of the SMPS analysis also revealed some bimodal particle size distributions [13]. The same three-stage pump was later used to compare ethane, ethylene, acetylene, and benzene/ethylene premixed flames [14]. The sampling apparatus was modified to perform dilution immediately at the point of sampling while increasing the total dilution to $\sim 40,000$ and the bimodal size distribution was confirmed [15].

A number of different sooting turbulent non-premixed flames have also been investigated in recent years. One of the earliest ethylene turbulent diffusion flames to be studied for soot was developed by Kent and Honnery [16]. They used extinction measurements to collect information on soot volume fraction. Some twenty years later, D'Anna et al. followed up with LIF, LII, and scattering measurements on the two of these flames, where they found a correlation of fluorescence signals with the mixture fraction [17]. Ethylene/air flames with $\operatorname{Re}=13,500$ were investigated by thermophoretic sampling followed by TEM [18] as well as with scattering and extinction measurements [19], yielding valuable quantities such as soot volume fraction, primary particle diameter, and aggregate size and fractal dimension. More recently, flames have been investigated which adhere more rigidly to the standards of the Turbulent Non-premixed Flame (TNF) Workshop in terms of well-characterized boundary conditions; an important consideration for modelers. DLR-Stuttgart has investigated a lifted ethylene flame with an air coflow and $\mathrm{Re}=10,000$. Flow velocity, temperature, and soot volume fraction measurements were taken [20] as well as fluorescence of PAH and $\mathrm{OH}$ and Raman scattering measurements of fuel/air mixing prior to combustion [21]. Another lifted ethylene flame has been under investigation at the University of Adelaide. It is composed of ethylene, hydrogen, and nitrogen with $\mathrm{Re}=15,000$ and recent measurements have provided 
joint probability density functions of soot volume fraction and temperature [22]. There are piloted sooting turbulent non-premixed flames as well. One example is the Delft Flame III, which uses a pilot to stabilize a flame of Dutch/Adelaide natural gas, for which LII was used to produce PDFs of soot volume fraction at various axial distances from the burner [23]. Another example developed at Sandia uses a premixed ethylene/air pilot to ignite a turbulent flame of pure ethylene over a range of Reynolds numbers. The pilot temperature profile was measured with Rayleigh line imaging while OH-PLIF and LII were used to characterize the turbulent flame [24]. A JP-8 surrogate burning variant was also included in the study.

The most common diagnostics utilized in studying sooting turbulent non-premixed flames are nonintrusive techniques, especially LII and scattering/extinction. Despite the obvious advantages of nonintrusive techniques, there are drawbacks as well. The combination of light scattering and extinction is not able to provide the soot primary particle diameter, whereas the combination of LII and light scattering can make the determination of soot volume fraction, primary particle diameter and aggregate size possible [25]. However, LII and light scattering are appropriate only for detection of larger soot particles [7]. LII may be used for quantitative measurements of both soot volume fraction and primary particle size, but this relies upon the ability to accurately model the physical factors that influence the LII signal [26]. Stirn et al. found good agreement between measurements made with SMPS and LII on premixed laminar ethylene/air flames in conditions where both methods are applicable, but with LII it was not possible to distinguish signals from smaller particles in the presence of larger, more luminous particles [27]. To adequately characterize the particle size distribution over a wide range of particle diameters, an intrusive technique like SMPS may be required.

In this study, an attempt is made to measure the particle size distribution function in a sooting turbulent non-premixed using an SMPS. To the best of our knowledge, this is the first published case of this technique being used to characterize soot in a turbulent flame.

\section{Experiment}

\subsection{Burner geometry}

The turbulent flame investigated in this study uses a burner geometry similar to that described in Zhang et al. [24], which was recently one of the target turbulent flames in the International Sooting Flame 3 (ISF-3) Workshop. The central jet tube has an inner diameter of $D=3.2 \mathrm{~mm}$ and the jet is composed of $\mathrm{C}_{2} \mathrm{H}_{4} / \mathrm{N}_{2}$ with $\mathrm{N}_{2}$ composing $65 \%$ by volume. A pilot flame anchors the central flame and is composed of a premixture of $\mathrm{C}_{2} \mathrm{H}_{4} / \mathrm{Air}$ at an equivalence ratio of 0.9 . The pilot flame plate consists of 3 concentric rings of holes and sits $5 \mathrm{~mm}$ below the central tube exit plane. Gas flow rates are chosen such that the main jet Reynolds number is 20,000 and the pilot flame provides $6 \%$ of the heat release of the main jet. A co-annular flow of air with a diameter of $245 \mathrm{~mm}$ is provided to the flame at a velocity of $0.6 \mathrm{~m} / \mathrm{s}$. The entire burner is oriented vertically on a translation stage capable of sub-mm adjustments over a total stroke length of $1500 \mathrm{~mm}$.

\subsection{Sampling apparatus}

The sampling apparatus is composed of a $9.5-\mathrm{mm}$ stainless steel tube oriented perpendicular to the flame. The tube has a $500-\mu \mathrm{m}$ orifice drilled into it which is aligned with the flame centerline and draws small amounts of flame product into the tube. A mass flow controller 


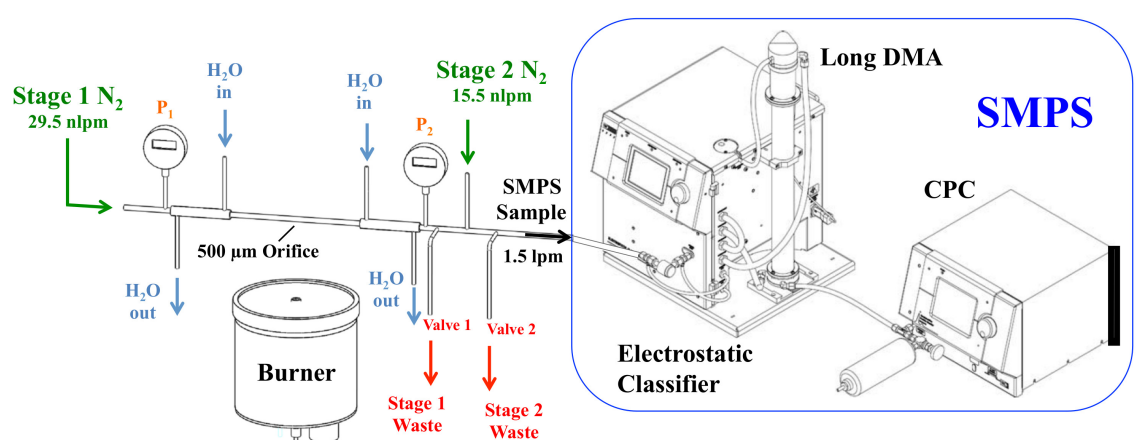

Fig. 1 SMPS sampling system with two stages of $\mathrm{N}_{2}$ dilution.

sets the flow rate of $\mathrm{N}_{2}$ used as the diluent in the sample tube. Pressure gauges are placed upstream and downstream of the orifice to estimate the pressure inside the tube at the orifice. A metering valve and vacuum pump are placed downstream of the second pressure gauge, allowing fine control of the pressure in the tube. Water-cooling is provided to the exterior surface of the sampling tube both upstream and downstream of the orifice.

In the second iteration of the apparatus, a second dilution stage is added downstream of the first stage to increase the amount of total sample dilution achievable. Similar to the first stage, a mass flow controller meters the amount of additional $\mathrm{N}_{2}$ allowed into the system after the majority of the sample gas from the first stage is purged. A second metering valve and vacuum pump are placed downstream of the second $\mathrm{N}_{2}$ input to allow control of the second stage dilution ratio. The total system has four devices for controlling the ratio of flame sample to diluent: two mass flow controllers and two valves. In practice, the first flow rate is kept constant at $29.5 \mathrm{nlpm}$ (normal liters per minute; reference $273.15 \mathrm{~K}, 101.325$ $\mathrm{kPa}$ ) and the second flow rate is kept constant at $15.5 \mathrm{nlpm}$. The first valve is used to control the dilution ratio in the first stage by controlling the pressure inside the tube at the orifice. The second valve controls the amount of sample from the first stage that is passed to the second stage. The entire two-stage dilution apparatus and SMPS are shown in Figure 1.

\subsection{Scanning mobility particle sizer}

The SMPS is composed of two separate instruments; the electrostatic classifier and the condensation particle condenser (CPC). In the classifier, particles in a polydisperse aerosol are first charged in the aerosol neutralizer and then separated in a differential mobility analyzer (DMA). The DMA applies a high voltage to a central rod and the resulting electric field separates the charged particles based on their electrical mobility. Only particles within a narrow range of electrical mobility exit the DMA sample flow outlet as a monodisperse aerosol. The selected particles are passed to the CPC where they form droplets in a condenser. The droplets then pass through a light beam and scatter light onto a photodetector, and the concentration is computed from the frequency of pulses of scattered light. The SMPS controls the size of the particle being counted by varying the voltage in the DMA. By scanning over a range of voltages, the concentrations of a wide range of particle diameters in the sample flow may be collected in a short period of time. The advantage of using the SMPS is that 
it can yield the entire soot particle size distribution with no manipulation of the sample and without relying on assumptions about the optical properties of the soot.

The SMPS used in this study is manufactured by TSI ${ }^{\circledR}$ and consists of a Model 3080 electrostatic classifier with the Model 3085 Nano DMA and a Model 3775 CPC. The sample flow rate is set to $1.5 \mathrm{lpm}$ and the sheath flow rate is $15.0 \mathrm{lpm}$. Under these conditions, the SMPS is capable of scanning over a range of particle diameters from $2 \mathrm{~nm}$ up to almost 70 $\mathrm{nm}$. Multiple scan times were investigated, and little difference was found in the results. A scan time of 120 seconds is used for all conditions.

\subsection{Procedure}

Before proceeding with in-flame measurements over a range of distances from the nozzle exit, it is necessary to ensure that errors in the sampling and measurement process are reduced as much as possible. One potential source of error is from deposition of soot on the orifice which can cause the measured particle concentration to be time-dependent. The SMPS takes time-averaged measurements, meaning that the time scale of the measurements (on the order of one-tenth of a second) is much greater than the time scale of turbulent fluctuations in the flame. Ideally, the dilution ratio of nitrogen to nanoparticles from the flame in the sampling tube will be constant during the 120-second SMPS scan time. However, if an appreciable amount of soot is deposited on the orifice wall during the scan, then measurements at the beginning of the scan will correspond with a different (lower) dilution ratio than those at the end of the scan.

Early experiments with pure $\mathrm{C}_{2} \mathrm{H}_{4}$ flames indicated that soot deposition could occur very quickly and could completely clog the orifice in less than a minute. To ensure that clogging is not a source of error, measurements in the CPC mode are carried out. In this mode of operation, a single voltage is used instead of scanning over a range so that a specific particle diameter is observed as a function of time. Since a single particle size is selected during a CPC experiment and the timescale of the sampling rate is much longer than the timescale of turbulent fluctuation, the instrument should measure a roughly constant concentration as a function of time. During measurements, the sample probe and the gases expand due to heating and cause a rise in the sampling tube pressure. This is prevented by incrementally opening up the valve 1 continuously, while keeping valve 2 fixed. The rate of sample intake into the probe and consequently the dilution is thus kept constant for any desired reading on pressure gage $\mathrm{P}_{2}$. A particle size of $8 \mathrm{~nm}$ and time duration of 300 seconds is chosen. Eliminating the measurements in the initial 90 seconds, which is approximately the time taken to achieve steady-state operation, the particle concentration over the remaining 210 seconds is found to stay within a factor of two and no long-term decline is observed. Thus, the orifice did not get clogged during the data collection process.

An additional benefit of the CPC procedure is that a "wait time" of 90 seconds, during which the flame and sampling system reach a steady state, is determined. As a result, all subsequent SMPS measurements are initiated at least 90 seconds after the flame is ignited. Furthermore, CPC studies prove that keeping a constant pressure reading on $\mathrm{P}_{2}$ by continuously adjusting valve 1 is sufficient to maintain a constant dilution ratio during the experiment and continuous operation of valve 2 is not necessary.

A second potential source of error is from particle-particle coagulation in the sampling line. When a significant amount of post-flame coagulation occurs, the measured particle concentrations are no longer representative of the true concentrations in the flame. Instead, the measured concentrations of smaller particles are artificially low while those of larger 
particles are artifically high, thus distorting the observed particle size distribution function (PSDF). Coagulation of soot particles in between the point of sampling and detection in the CPC must be eliminated or its effects on the PSDF must be estimated. The most effective way to eliminate coagulation is to ensure sufficient dilution of the sample, which effectively "freezes" the reactions. The dilution ratio is controlled by opening and closing the valves.

To test the effects of coagulation along the sampling line and to optimize the valve settings, a parametric study over a range of pressures and valve 2 settings is conducted at the $x / D$ of peak total soot concentration. Note that the $\mathrm{P}_{2}$ reading is an indirect measure of the actual orifice pressure but it has been shown to determine the dilution ratio in the first sampling stage and may consequently be seen as a proxy for that measurement. The actual orifice pressure is unknown and is not measured in our experiments. In all cases, the $\mathrm{P}_{2}$ reading is maintained below atmospheric pressure to ensure that sample gases are being drawn from the flame through the orifice. The results of the parametric studies (see section 3.1) indicate that there are a number of settings for which coagulation is apparently eliminated.

Additional sources of error are handled during the processing of the raw data collected by the CPC. The AIM software developed by $\mathrm{TSI}^{\circledR}$ has some data correction algorithms. The diffusion loss algorithm corrects for particles lost as they diffuse to the tubing walls within the SMPS instrument. For large particle diameters, a multiple charge algorithm is used to correct for particles that have higher mobility in the DMA due to multiple charges rather than aerodynamic considerations (drag) based on the true particle diameter. A diffusion loss correction is applied to the processed SMPS data to account for losses in the sampling tube upstream of the impactor as well. The effects of these corrections are quantified in section 3.3.

Using an appropriate combination of pressure and valve 2 position, SMPS scans are performed along the flame centerline at multiple axial locations in increments of $\Delta x / D=5$. To prevent accumulation of soot on the sampling tube and around the orifice, the flame is present only during the scans and the orifice is regularly cleaned with wire between scans. Generally, two consecutive 120 -second scans are performed at a given location and then the orifice is cleaned before moving to the next location. After post-processing, multiple data sets from the Nano-DMA for each $x / D$ are merged to create soot PSDFs as a function of height from the nozzle exit.

\section{Results and Discussion}

\subsection{Dilution effects}

To eliminate the possibility of coagulation in the sample tube, a parametric study of different valve settings was used to investigate the effect of dilution on the measured particle concentration probability density functions (PDFs). Such tests were performed on both a $\operatorname{Re}_{D}=$ 10,000 flame and the $\mathrm{Re}_{D}=20,000$ flame, both composed of $\mathrm{C}_{2} \mathrm{H}_{4}$ with $65 \% \mathrm{~N}_{2}$ dilution. The study on the $\operatorname{Re}_{D}=10,000$ flame was more extensive because it was the first flame to be investigated and it exhibits generally higher total particle concentrations than the $\operatorname{Re}_{D}=$ 20,000 flame. It was believed that a set of conditions that work on the higher sooting flame would work for the other flame as well, and this is shown to be true.

For each condition in the study, a different combination of valve settings was used. By opening the first valve, a lower negative pressure $(P$, measured by the second pressure gage $\mathrm{P}_{2}$ downstream of the orifice) is achieved and more sample is drawn from the flame. 
Table 1 Total volume concentration $\left(V, \mathrm{~nm}^{3} / \mathrm{cm}^{3}\right)$ for each pressure $(P, \mathrm{kPa})$ and valve 2 setting in the parametric dilution ratio study for $\operatorname{Re}_{D}=10,000$.

\begin{tabular}{|c|c|c|c|c|}
\hline & $\begin{array}{c}\text { Two-Stage, } \\
\text { Low }\end{array}$ & $\begin{array}{c}\text { Two-Stage, } \\
\text { Medium }\end{array}$ & $\begin{array}{c}\text { Two-Stage, } \\
\text { High }\end{array}$ & $\begin{array}{c}\text { Single- } \\
\text { Stage }\end{array}$ \\
\hline$P=-0.15$ & $3.3 \times 10^{8}$ & $2.1 \times 10^{9}$ & $1.7 \times 10^{9}$ & $6.6 \times 10^{9}$ \\
\hline$P=-0.30$ & $1.9 \times 10^{9}$ & $9.4 \times 10^{9}$ & $1.6 \times 10^{10}$ & $8.5 \times 10^{10}$ \\
\hline$P=-0.60$ & - & $2.3 \times 10^{10}$ & $5.5 \times 10^{10}$ & $3.0 \times 10^{11}$ \\
\hline$P=-1.20$ & - & - & $1.0 \times 10^{11}$ & $9.8 \times 10^{11}$ \\
\hline
\end{tabular}

Four values of $P$ were chosen for the study. Similarly, by opening the second valve, more sample is drawn from the first stage. Three valve settings (low, medium, high) were chosen and an additional condition was added in which there was only one stage of dilution. Of the sixteen possible pressure/valve combinations in the study, only thirteen were achievable due to limitations in the capacity of the vacuum pumps. For each test, two consecutive 120 -second scans were performed. The valve combinations and the resulting total volume concentrations achieved are reported in Table 1.

We know that total number concentrations are affected by coagulation, so to get a sense of the range of dilution ratios covered in this study, we may instead examine the range of total volume concentrations. This requires a few assumptions. First, we can neglect differences in thermal expansion of the diluent amongst the thirteen cases, which should be minor due to the water-cooled sections of tubing. Second, we assume that volume contributions from particles with diameters larger than $64 \mathrm{~nm}$ are not significant. A crude analysis of the existing data indicates that the inclusion of larger particles would increase the total volume of soot by no more than a few percent in the least diluted case and far less than this in all other cases. Lastly, we assume that total volume concentration is conserved, even in the presence of coagulation in the sampling line. This is true if soot particles are spherical, regardless of the processes that formed them, meaning the mobility diameter as measured by the SMPS can be used to calculate the volume of the soot particles. Since this assumption is implied in the act of using the SMPS to measure soot particle distributions in the first place, we will maintain this assumption for the sake of this argument. Under these assumptions, the range of total volume concentrations is exactly representative of the range of dilution ratios covered in this study.

The values in Table 1 indicate that increasing the pressure (increasing the dilution ratio in the first stage) and shifting from single-stage to the lowest two-stage dilution (increasing the dilution ratio in the second stage) both result in decreasing values of $V$ as expected. The ratio of $V$ from the least diluted case $(P=-1.20 \mathrm{kPa}$, single-stage) to the most diluted case $(P=-0.15 \mathrm{kPa}$, low $)$ is almost 3,000 . We may safely assume that the dilution ratio even in the least diluted case is greater than 3, meaning the most diluted case is on the order of magnitude of 10,000 or more, which is a reasonable dilution ratio based on previous studies on laminar flames [9]. It should be re-emphasized that this is a very rough estimation and further investigation is required to assess the true dilution ratio at which our measurements were taken. Nonetheless, we surmise that a sufficiently large range of dilution ratios is covered and that $V$ may be used as a proxy for the dilution ratio. To avoid confusion, it is worth emphasizing that $V$ is a quantity measured by the SMPS and thus represents the total volume concentration in the sample, not the concentration at the sampling location in the flame.

A process for ensuring adequate dilution by comparing soot PSDFs at different levels of dilution was demonstrated by Zhao et al. [8]. Coagulation tends to distort nanoparticle PDFs as measured in the SMPS. The elimination of coagulation effects through sufficient 


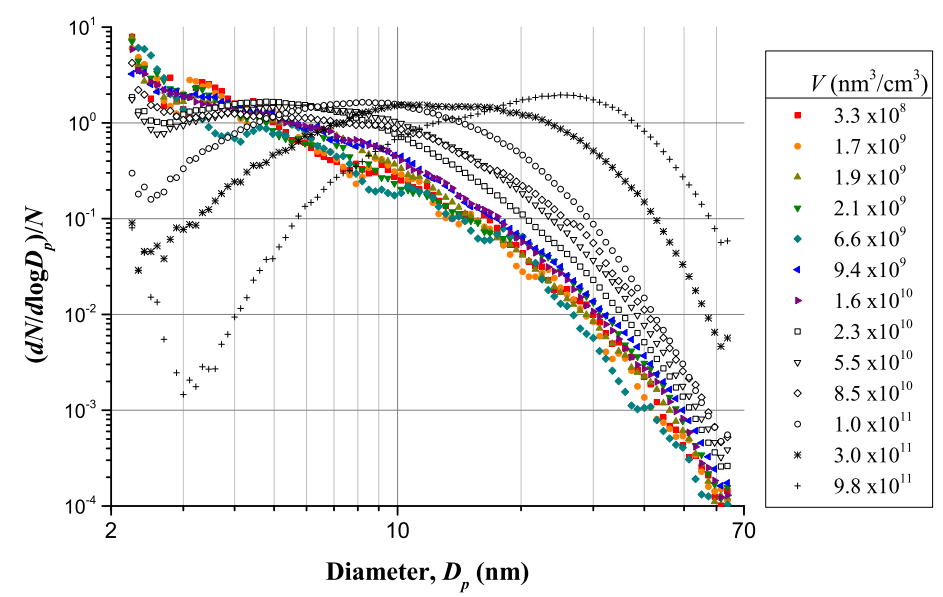

Fig. 2 Nanoparticle number concentration PDFs for all cases in a dilution ratio study. Measurements were taken along the flame centerline at $x / D=60$ in a $\operatorname{Re}_{D}=10,000$ flame.

dilution in the sampling line should result in a number concentration PDF that is invariant with further increases in the dilution ratio (provided the detection limit of the instrument is not exceeded). Number concentration PDFs for all thirteen cases are given in Figure 2. The corresponding volume concentration PDFs for the same cases are given in Figure 3. There is a remarkable similarity in the distributions for the seven (colored) cases with the lowest total volume concentrations, and thus the highest dilution ratios, whereas in the other six cases, a severe divergence in the shape of the PDFs is revealed. Without exception, as the total volume concentration, $V$, increases, the distribution exhibits higher concentrations of large nanoparticles relative to the concentration of small nanoparticles. This behavior strongly suggests coagulation of nanoparticles in the sampling line in the six least diluted cases.

The trends in the distributions may be better understood by examining the moments of the PDFs. In Figure 4 the mean and skewness of the number concentration distributions are plotted as a function of the corresponding total volume concentration for each condition in the parametric study. At high total volume concentrations, the mean tends to decrease as the total volume concentration decreases. However, below a certain value of $V$, this trend flattens out. This critical total volume concentration, $V_{\text {crit }}$, has a value of approximately $2.0 \times 10^{10} \mathrm{~nm}^{3} / \mathrm{cm}^{3}$. The trend in skewness of the distributions is not as clear. The change in skewness as a function of $V$ is almost linear for cases above the critical value. Due to the large variability in the skewness data for the cases below the critical value, the conclusion that these cases exhibit the same distribution is perhaps debatable. Nonetheless, for the seven cases for which $V<V_{\text {crit }}$, the mean diameter is approximately $4.3 \mathrm{~nm}$ and the skewness is greater than 3. For these cases, the sample is considered to be sufficiently diluted.

A similar, but less exhaustive, study was performed for the $\mathrm{Re}_{D}=20,000$ flame. Only five conditions were tested. The settings and the corresponding total volume concentrations are given in Table 2. Number concentration PDFs for all five cases are given in Figure 5. The corresponding volume concentration PDFs for the same cases are given in Figure 6. As expected from the other study, the cases with the lowest total volume concentrations (and highest dilution ratios) exhibit very similar PDFs, indicating that the effects of coagulation 


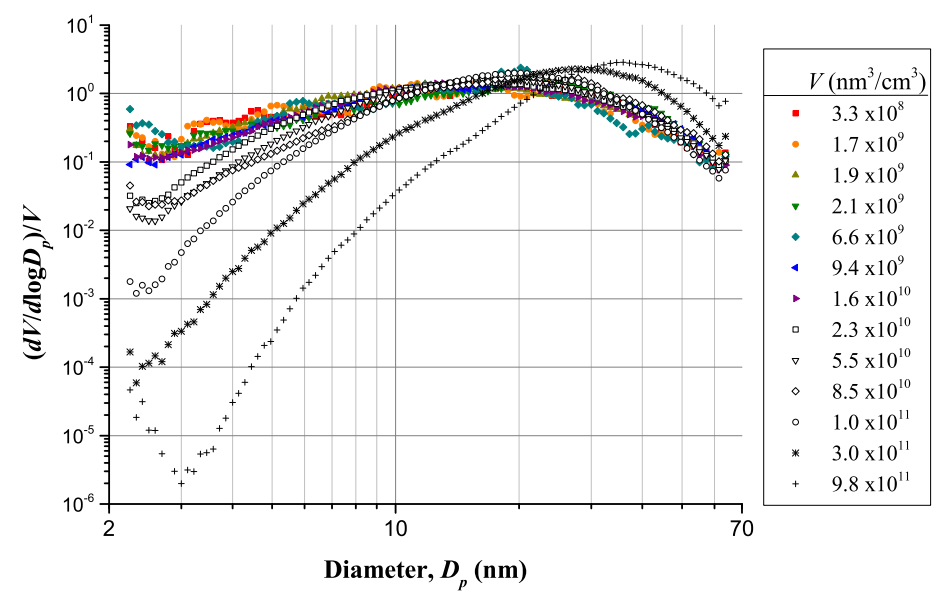

Fig. 3 Nanoparticle volume concentration PDFs for all cases in a dilution ratio study. Measurements were taken along the flame centerline at $x / D=60$ in a $\operatorname{Re}_{D}=10,000$ flame.

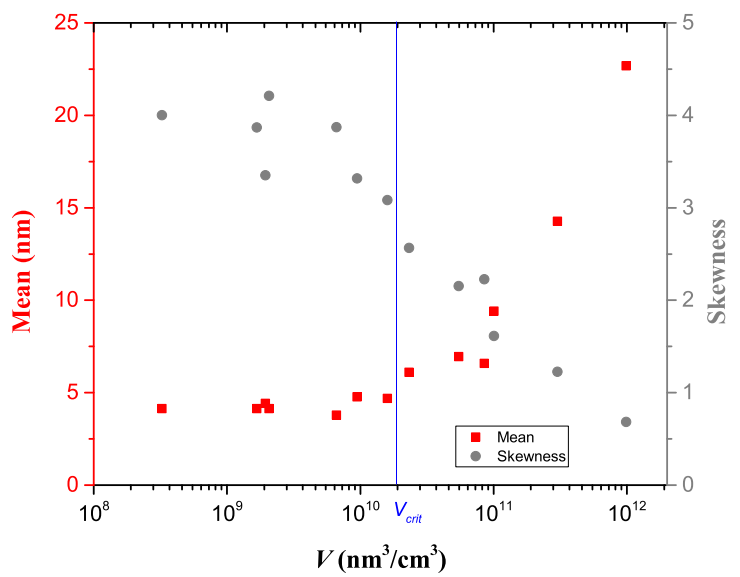

Fig. 4 Mean and skewness of the number concentration PDFs. Values are plotted as a function of the total volume concentration for each set of conditions in the $\operatorname{Re}_{D}=10,000$ dilution ratio parametric study.

are minimized and the samples are sufficiently diluted. The critical total volume concentration for this flame, $V_{\text {crit }} \approx 2.0 \times 10^{9} \mathrm{~nm}^{3} / \mathrm{cm}^{3}$, is an order of magnitude lower than the value from the $\operatorname{Re}_{D}=10,000$ study. For the four cases for which $V<V_{\text {crit }}$, the mean diameter is approximately $3.1 \mathrm{~nm}$ and the variance is approximately $1.3 \mathrm{~nm}^{2}$.

Results of these parametric studies show that it is possible to sample from a sooting turbulent non-premixed flame and analyze the resulting samples in a SMPS, but it is important to characterize the effect of the sampling method on the results. In this case, a two-stage dilution system was a significant improvement over a single-stage dilution system. Looking at Table 1 and Table 2, it is evident that even small changes in the pressure at the orifice 
Table 2 Total volume concentration $\left(V, \mathrm{~nm}^{3} / \mathrm{cm}^{3}\right)$ for each pressure $(P, \mathrm{kPa})$ and valve 2 setting in the parametric dilution ratio study for $\operatorname{Re}_{D}=20,000$.

\begin{tabular}{|c|c|c|}
\hline & $\begin{array}{c}\text { Two-Stage, } \\
\text { Low }\end{array}$ & $\begin{array}{c}\text { Two-Stage, } \\
\text { Medium }\end{array}$ \\
\hline$P=-0.15$ & $2.8 \times 10^{7}$ & $9.8 \times 10^{7}$ \\
\hline$P=-0.30$ & $2.9 \times 10^{8}$ & $1.2 \times 10^{9}$ \\
\hline$P=-0.60$ & - & $4.9 \times 10^{9}$ \\
\hline
\end{tabular}

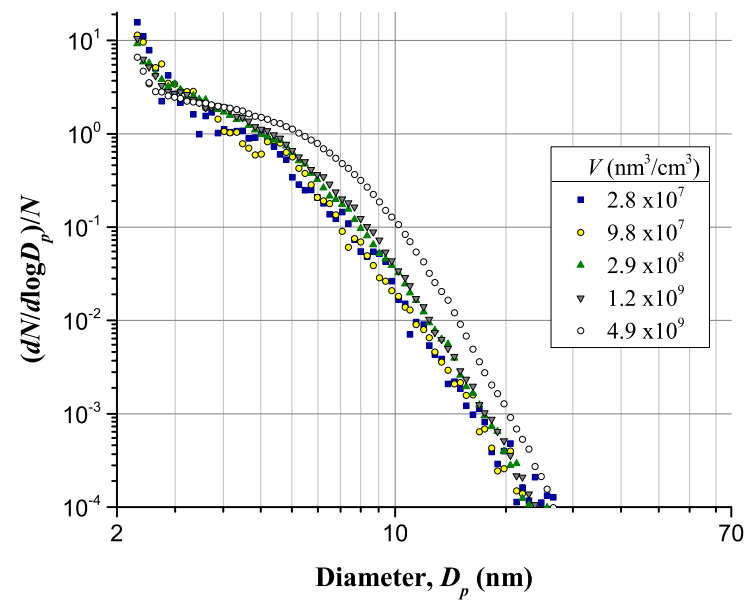

Fig. 5 Nanoparticle number concentration PDFs for all cases in a dilution ratio study. Measurements were taken along the flame centerline at $x / D=70$ in a $\operatorname{Re}_{D}=20,000$ flame.

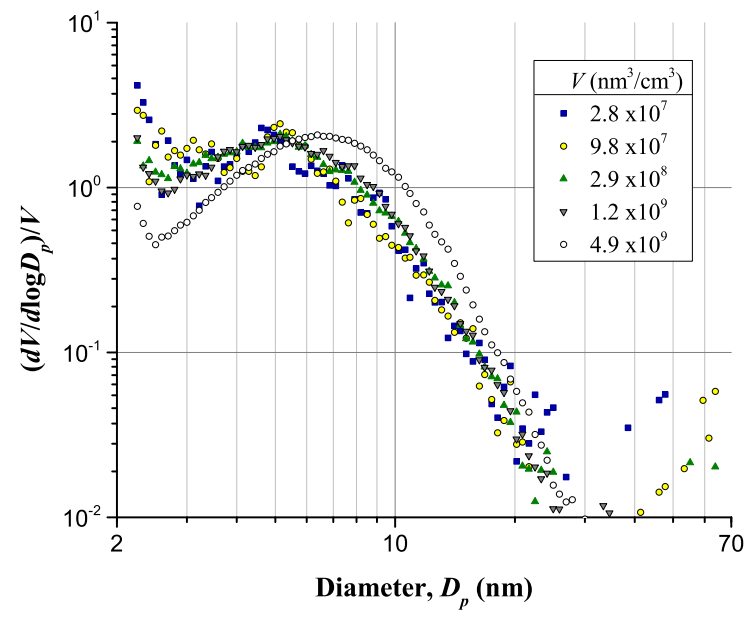

Fig. 6 Nanoparticle volume concentration PDFs for all cases in a dilution ratio study. Measurements were taken along the flame centerline at $x / D=70$ in a $\operatorname{Re}_{D}=20,000$ flame. 
Table 3 Mean $(\bar{x})$, geometric mean $\left(\bar{x}_{g}\right)$, variance $\left(\sigma^{2}\right)$, and geometric standard deviation $\left(\sigma_{g}\right)$ at multiple $x / D$ heights.

\begin{tabular}{|c|c|c|c|c|}
\hline$x / D$ & $\bar{x}(\mathrm{~nm})$ & $\bar{x}_{g}(\mathrm{~nm})$ & $\sigma^{2}\left(\mathrm{~nm}^{2}\right)$ & $\sigma_{g}(\mathrm{~nm})$ \\
\hline 60 & 2.8 & 2.7 & 0.4 & 1.2 \\
\hline 65 & 3.1 & 3.0 & 1.0 & 1.3 \\
\hline 70 & 3.5 & 3.3 & 2.0 & 1.4 \\
\hline 75 & 4.0 & 3.7 & 3.5 & 1.5 \\
\hline 80 & 4.4 & 4.0 & 5.5 & 1.5 \\
\hline 85 & 4.8 & 4.1 & 9.7 & 1.7 \\
\hline 90 & 5.6 & 4.6 & 15.8 & 1.8 \\
\hline
\end{tabular}

can yield large differences in dilution ratio. Changes in the second valve setting have a less pronounced effect. However, the addition of a second stage of dilution allowed the dilution ratio to increase by at least an order of magnitude. It is also worth noting that although the dilution ratio is greatest for the highest pressure setting, this was not the ideal setting for taking measurements. One reason is that because the pressure is so close to atmospheric pressure, small fluctuations in orifice pressure during the experiment (which are impossible to eliminate) can create artifical oscillations in the results. Another reason is that it is best to keep the dilution ratio below the critical value but not too far below, as there is more uncertainty in the measurement of highly diluted samples.

\subsection{Particle size distribution functions}

Given consistent concentration measurements and sufficient dilution, particle sizing was carried out at multiple axial locations in the flame. All measurements were taken along the flame centerline at increments of $\Delta x / D=5$. Multiple scans were taken and averaged for the final results. It was observed that in all cases, scan-to-scan variation was negligible. The resulting PSDFs are given in Figure 7. In the figure, a progressive shift from smaller particles to larger particles is seen as one moves away from the nozzle exit. The geometric mean particle diameter increases monotonically as a function of $x / D$. See Table 3 . At higher axial locations, the size distribution tends to flatten out in the range of 4-9 $\mathrm{nm}$. It is noted that at no location in the flame are particle diameters above the upper limit of the Nano-DMA $(\sim 70 \mathrm{~nm})$ measured in any significant concentration. Although a different DMA with higher range was available for use, it was not necessary for this flame.

Due to the difficulties in measuring soot particle size distribution functions in turbulent flames, there is little data with which to compare these results. One numerical study of relevance was performed by Netzell et al. [28]. A rim-stabilized turbulent ethylene flame issuing from a tube with a diameter of $3.1 \mathrm{~mm}$ and at a velocity of $24.5 \mathrm{~m} / \mathrm{s}$ was used for the study. A detailed chemical mechanism with a soot model was used with a sectional method to generate particle size distribution functions along the centerline at multiple axial locations in the flame. The trends appear to be quite similar to the results obtained in this study, in that the distribution is neither bimodal nor log-normal and the mean particle diameter continuously increases further from the nozzle.

A more recent numerical study by Abdelgadir et al. [29] may yield more insight into the nature of the observed PSDFs. In this study, a turbulent non-premixed $n$-heptane flame with a jet Reynolds number of 15,000 was investigated via direct numerical simulation (DNS). The results were post-processed using a Monte Carlo method and evolution of soot along certain 


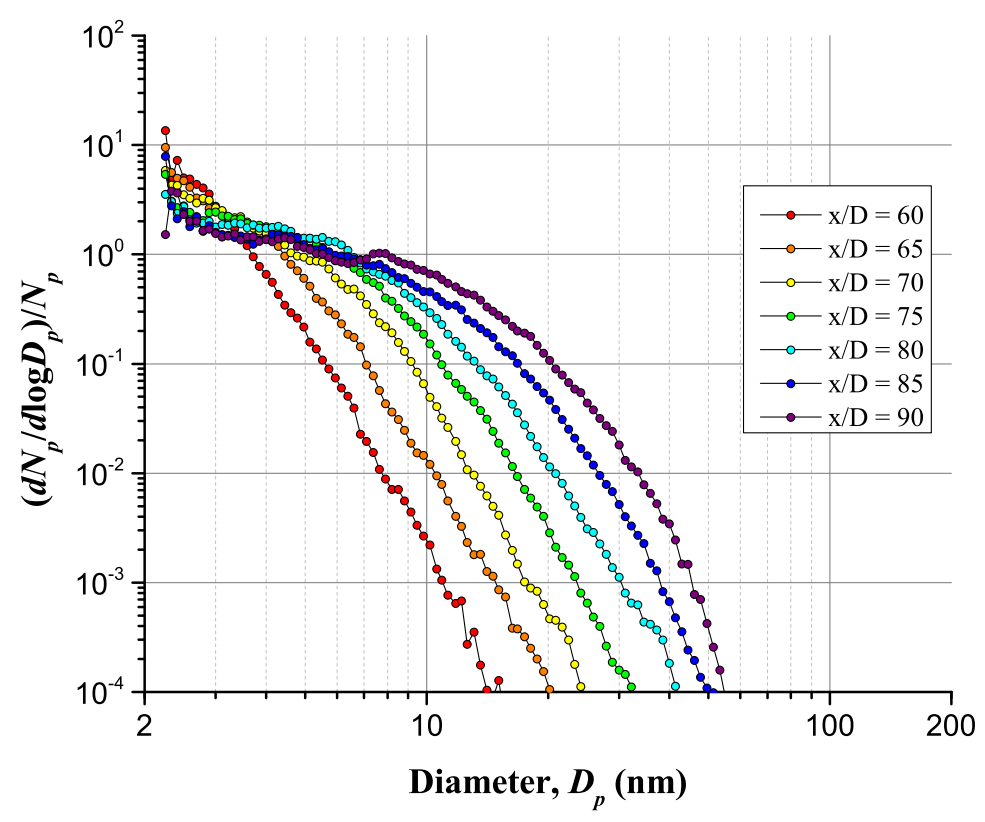

Fig. 7 Centerline soot PSDFs at multiple $x / D$ heights in a $\operatorname{Re}_{D}=20,000$ flame.

Lagrangian trajectories was reported. The findings indicate that instantaneous soot PSDFs at any given location on the centerline (at a given time in the simluation) may take on a wide range of unimodal or bimodal shapes. However, the average PSDF of these distributions exhibits a high value at low particle diameters with a monotonically decreasing probability as the particle diameter increases. In light of these results, the PSDFs presented in Figure 7 may in fact represent the effect of many different, more familiar (from work in laminar premixed flames) distributions integrated over time.

\subsection{Uncertainty and error}

Diffusion losses inside the instrument and errors from multiply charged particles were corrected for by the SMPS software algorithm provided by TSI ${ }^{\circledR}$. The magnitude of the corrections applied by both algorithms is a function of the particle diameter. For the diffusion loss algorithm, the effect is greatest on the smallest diameter particles. For concentrations of particles in the $2.25 \mathrm{~nm}$ bin, the correction is over $130 \times$. The magnitude of this correction decreases exponentially with increasing diameter: it is about $11 \times$ for the $4.00 \mathrm{~nm}$ bin, and is less than $2 \times$ for diameters greater than $13.00 \mathrm{~nm}$. The multiple charge algorithm applies only to particles with very large diameters (approaching $100 \mathrm{~nm}$ ) and is essentially 1 for all cases reported here. Losses upstream of the instrument were accounted for as well during post-processing of the SMPS data, but this correction is relatively minor, resulting in a $2 \times$ increase for only the smallest $(<2.50 \mathrm{~nm})$ particles. The data collected has been presented in its entirety for the sake of completeness, however, because of the extreme corrections to 
the raw data, some caution should be exercised when considering the data, especially in the range below $4 \mathrm{~nm}$.

In general, the uncertainty in these measurements is a strong function of the particle number concentration. The accuracy of the concentration reading is improved by increasing the number of particles detected by the CPC. This works in our favor because the conditions with low particle concentrations, and therefore greater uncertainty, also contribute least to the PDFs. Further, for this purposes of this study, we are only concerned with the particle concentrations at a given diameter relative to other concentrations (because we are only evaluating the PDFs) so any instrumental uncertainties that could impact the total number concentration (such as uncertainties in flow rate) are neutralized. On the other hand, the counting efficiency for our CPC decreases sharply with decreasing particle diameter, below $10 \mathrm{~nm}$. The counting efficiency at $4 \mathrm{~nm}$ is less than $50 \%$. The software tends to compensate for this loss in efficiency and may over-correct the data. Regarding the measurement error, for every condition, two measurements were made. The ratio of the higher value to the lower value rarely exceeds 2 . The relative error is close to $50 \%$ for a few samples and remains below $10 \%$ for the vast majority of samples taken. As discussed previously, it is important to keep in mind that one limitation of the SMPS is that it actually measures particle mobility diameter and soot particles are not perfectly spherical. Lastly, as with all instrusive techniques, there is inevitably some flow disturbance created by the probe itself.

\section{Conclusion}

It has been shown that the scanning mobility particle sizer is a useful tool for soot characterization not only for laminar flames, but also for highly diluted turbulent flames. Care must be taken to ensure that measured particle concentrations are not fluctuating with time and that coagulation effects in the sample line are eliminated as much as possible. In this case, a second dilution stage was necessary for the latter condition. Centerline soot PSDFs were measured for a turbulent non-premixed nitrogen-diluted ethylene flame with $\operatorname{Re}_{D}=20,000$. The size distributions reveal that the mean nanoparticle diameter increases with distance from the nozzle. In all cases, the number concentration peaks for particle diameters below 4 $\mathrm{nm}$ and the concentration decreases monotonically with increasing particle diameter.

Acknowledgements The research reported in this publication was supported by funding from King Abdullah University of Science and Technology (KAUST).

\section{References}

1. Peter R. Buseck and Mihály Pósfai. Airborne minerals and related aerosol particles: Effects on climate and the environment. Proc. Natl. Acad. Sci. U. S. A., 96(7):3372-3379, 1999.

2. Thomas J. Grahame, Rebecca Klemm, and Richard B. Schlesinger. Public health and components of particulate matter: The changing assessment of black carbon. J. Air Waste Manage. Assoc., 64(6):620$660,2014$.

3. Ming-Tung Chuang, Chung-Te Lee, Charles C.-K. Chou, Neng-Huei Lin, Guey-Rong Sheu, Jia-Lin Wang, Shuenn-Chin Chang, Sheng-Hsiang Wang, Kai Hsien Chi, Chea-Yuan Young, Hill Huang, HorngWen Chen, Guo-Hau Weng, Sin-Yu Lai, Shao-Peng Hsu, Yu-Jia Chang, Jia-Hon Chang, and XyueChang Wu. Carbonaceous aerosols in the air masses transported from Indochina to Taiwan: Long-term observation at Mt. Lulin. Atmos. Environ., 89:507-516, 2014.

4. Surabi Menon, James Hansen, Larissa Nazarenko, and Yunfeng Luo. Climate Effects of Black Carbon Aerosols in China and India. Science (80-. )., 297:2250-2253, 2002. 
5. Aneta Wierzbicka, Patrik T. Nilsson, Jenny Rissler, Gerd Sallsten, Yiyi Xu, Joakim H. Pagels, Maria Albin, Kai Österberg, Bo Strandberg, Axel Eriksson, Mats Bohgard, Kerstin Bergemalm-Rynell, and Anders Gudmundsson. Detailed diesel exhaust characteristics including particle surface area and lung deposited dose for better understanding of health effects in human chamber exposure studies. Atmos. Environ., 86:212-219, 2014.

6. Hai Wang. Formation of nascent soot and other condensed-phase materials in flames. Proc. Combust. Inst., 33:41-67, 2011.

7. Andrea D'Anna. Combustion-formed nanoparticles. Proc. Combust. Inst., 32:593-613, 2009.

8. Bin Zhao, Zhiwei Yang, Murray V. Johnston, Hai Wang, Anthony S. Wexler, Michael Balthasar, and Markus Kraft. Measurement and numerical simulation of soot particle size distribution functions in a laminar premixed ethylene-oxygen-argon flame. Combust. Flame, 133:173-188, 2003.

9. Bin Zhao, Zhiwei Yang, Jinjin Wang, Murray V. Johnston, and Hai Wang. Analysis of Soot Nanoparticles in a Laminar Premixed Ethylene Flame by Scanning Mobility Particle Sizer. Aerosol Sci. Technol., 37:611-620, 2003 .

10. Bin Zhao, Zhiwei Yang, Zhigang Li, Murray V. Johnston, and Hai Wang. Particle size distribution function of incipient soot in laminar premixed ethylene flames: effect of flame temperature. Proc. Combust. Inst., 30:1441-1448, 2005.

11. Aamir D. Abid, Nicholas Heinz, Erik D. Tolmachoff, Denis J. Phares, Charles S. Campbell, and Hai Wang. On evolution of particle size distribution functions of incipient soot in premixed ethylene-oxygenargon flames. Combust. Flame, 154:775-788, 2008.

12. Bin Zhao, Kei Uchikawa, and Hai Wang. A comparative study of nanoparticles in premixed flames by scanning mobility particle sizer, small angle neutron scattering, and transmission electron microscopy. Proc. Combust. Inst., 31:851-860, 2007.

13. M. Matti Maricq, Stephen J. Harris, and Joseph J. Szente. Soot size distributions in rich premixed ethylene flames. Combust. Flame, 132:328-342, 2003.

14. M. Matti Maricq. A comparison of soot size and charge distributions from ethane, ethylene, acetylene, and benzene/ethylene premixed flames. Combust. Flame, 144:730-743, 2006.

15. M. M. Maricq. Size and charge of soot particles in rich premixed ethylene flames. Combust. Flame, 137:340-350, 2004.

16. J. H. Kent and D. Honnery. Soot and Mixture Fraction in Turbulent Diffusion Flames. Combust. Sci. Technol., 54:383-398, 1987.

17. Andrea D'Anna, Mario Commodo, Santo Violi, Cristophe Allouis, and John Kent. Nano organic carbon and soot in turbulent non-premixed ethylene flames. Proc. Combust. Inst., 31:621-629, 2007.

18. Bing Hu, Bo Yang, and Umit O. Koylu. Soot measurements at the axis of an ethylene/air non-premixed turbulent jet flame. Combust. Flame, 134:93-106, 2003.

19. Bo Yang and Umit O. Koylu. Detailed soot field in a turbulent non-premixed ethylene/air flame from laser scattering and extinction experiments. Combust. Flame, 141:55-65, 2005.

20. M. Köhler, K. P. Geigle, W. Meier, B. M. Crosland, K. A. Thomson, and G. J. Smallwood. Sooting turbulent jet flame: characterization and quantitative soot measurements. Appl. Phys. B Lasers Opt., 104:409-425, 2011.

21. Markus Köhler, Klaus-Peter Geigle, Thomas Blacha, Peter Gerlinger, and Wolfgang Meier. Experimental characterization and numerical simulation of a sooting lifted turbulent jet diffusion flame. Combust. Flame, 159:2620-2635, 2012.

22. S. M. Mahmoud, G. J. Nathan, P. R. Medwell, B. B. Dally, and Z. T. Alwahabi. Simultaneous planar measurements of temperature and soot volume fraction in a turbulent non-premixed jet flame. Proc. Combust. Inst., 35:1931-1938, 2015.

23. N. H. Qamar, Z. T. Alwahabi, Q. N. Chan, G. J. Nathan, D. Roekaerts, and K. D. King. Soot volume fraction in a piloted turbulent jet non-premixed flame of natural gas. Combust. Flame, 156:1339-1347, 2009.

24. Jiayao Zhang, Christopher R. Shaddix, and Robert W. Schefer. Design of "model-friendly" turbulent non-premixed jet burners for C2+ hydrocarbon fuels. Rev. Sci. Instrum., 82:074101, 2011.

25. D. R. Snelling, O. Link, K. A. Thomson, and G. J. Smallwood. Measurement of soot morphology by integrated LII and elastic light scattering. Appl. Phys. B Lasers Opt., 104:385-397, 2011.

26. C. Schulz, B. F. Kock, M. Hofmann, H. A. Michelsen, S. Will, B. Bougie, R. Suntz, and G. Smallwood. Laser-induced incandescence: recent trends and current questions. Appl. Phys. B Lasers Opt., 83:333$354,2006$.

27. R. Stirn, T. Gonzalez Baquet, S. Kanjarkar, W. Meier, K. P. Geigle, H. H. Grotheer, C. Wahl, and M. Aigner. Comparison of Particle Size Measurements with Laser-Induced Incandescence, Mass Spectroscopy, and Scanning Mobility Particle Sizing in a Laminar Premixed Ethylene/Air Flame. Combust. Sci. Technol., 181:329-349, 2009. 
28. Karl Netzell, Harry Lehtiniemi, and Fabian Mauss. Calculating the soot particle size distribution function in turbulent diffusion flames using a sectional method. Proc. Combust. Inst., 31:667-674, 2007.

29. Ahmed Abdelgadir, Marco Lucchesi, Antonio Attili, and Fabrizio Bisetti. Investigation of soot morphology and particle size distribution in a turbulent nonpremixed flame via Monte Carlo simulations. In Proc. Eur. Combust. Meet., Budapest, Hungary, 2015. 